MINI-SYMPOSIUM

\title{
Acute pericardial disease: approach to the aetiologic diagnosis
}

\author{
G Permanyer-Miralda
}

Heart 2004;90:252-254. doi: 10.1136/hrt.2003.024802

\begin{abstract}
A cute pericardial disease includes acute pericarditis (friction rub, or characteristic pain and ECG) and cardiac tamponade. The strategy for its aetiologic diagnosis can be quite simple, because it is either associated with other conditions (such as myocardial infarction, collagen vascular disease, uraemia, or neoplasia) which give the correct clue to aetiology or, when presenting in isolation in developed countries, it is so called idiopathic and usually self limited in most cases. However, in comparatively few instances acute pericardial disease presenting in isolation may be caused by specific treatable diseases (mostly tuberculosis or neoplasia). It may then raise considerable diagnostic problems. Recent developments, such as pericardioscopy, ${ }^{12}$ and classical procedures such as pericardiocentesis or pericardial biopsy, may appear to be helpful; however, judicious use of invasive diagnostic procedures should always imply a systematic diagnostic reasoning based on the prevalence of specific diagnoses. Although acute pericardial disease can be caused by a vast array of agents or conditions, in immunologically competent patients from the western world most cases unassociated with apparent medical or surgical conditions are secondary to viral infection or the immunological response to it (in about $85 \%$ of cases in studies in Spain, for example)..$^{3-5}$ However, in other parts of the world, some of these causes may have a different prevalence that may accordingly lead to a modified diagnostic approach. Tuberculosis is a case in point. ${ }^{6}$ In affluent countries it is usually rare in immunologically competent people. However, in developing countries or in immunologically compromised patients, it may be a comparatively common pericardial disease, although its true prevalence is unknown. Different prevalences and different patient populations may justify different diagnostic approaches.
\end{abstract}

\section{ARE THERE CLINICAL CLUES TO AETIOLOGIC DIAGNOSIS?}

It would be useful if the different patterns of clinical presentation were sensitive or specific for given aetiologies. This is the case in most instances, but not invariably so. As previously stated, in patients with acute pericarditis and features suggesting associated disease the likelihood of this being the cause for the pericardial syndrome is very high. ${ }^{7}$ Therefore, associated disease should be investigated accordingly.

In the immunologically competent patient presenting with classically acute pericarditis, the probability of acute idiopathic pericarditis is about $90 \%,{ }^{3}$ this probability being even greater when clinical features subside in the following few days. No further studies are needed in such cases. Subsequent relapses are also highly specific for idiopathic pericarditis.

Tamponade in the context of acute pericarditis is a more complex problem. In a study of our group, ${ }^{3}$ tamponade was significantly more common in patients with tuberculous or purulent pericarditis or neoplasia, but the high prevalence of acute idiopathic pericarditis made it the leading cause in absolute terms (table 1). However, in tamponade without findings of inflammation (chest pain or rub) we have demonstrated $^{7}$ a likelihood ratio of 3.0 for neoplasia, although other aetiologies should be considered as well.

Other features such as a sustained clinical course (for example, over three weeks) also increase the likelihood of specific disease. However, in our part of the world, ${ }^{3}$ the predominance of idiopathic disease in any presentation of acute pericarditis and no associated conditions may be taken as a clinical rule. An exception is tamponade with massively haemorrhagic effusion, often but not always caused by malignancy. ${ }^{8}$ In addition, purulent pericarditis should always be considered in predisposing diseases (such as pleural empyema or mediastinal infection), even in the absence of definite features of pericarditis or tamponade. ${ }^{9}$

Thus, in only comparatively few patients with acute pericardial disease (mainly those with haemodynamic compromise or protracted course) will specific pericardial investigations be needed for aetiologic diagnosis.

\section{DIAGNOSTIC YIELD OF PERICARDIOCENTESIS, PERICARDIAL BIOPSY, AND PERICARDIOSCOPY}

Since 1977 our group has undertaken several cohort studies of patients with pericardial disease. ${ }^{39} 9^{10}$ A key finding of these studies ${ }^{3}$ was that the diagnostic yield of either pericardiocentesis or subxiphoid pericardial drainage with biopsy were significantly much greater when performed on patients with cardiac tamponade than when indicated for purely diagnostic purposes (for example 35\% $\vee 5 \%{ }^{3}$ ). In addition, we showed that persistent mild or moderate effusion was not by itself an indication for routine invasive diagnostic studies, as no late events associated with effusion were observed and effusion resolved in most cases. ${ }^{10}$ Suggested alternative diagnoses may not be clinically relevant. ${ }^{11}$ Probably, the most appropriate approach represents a compromise between performing too many unnecessary invasive studies and missing too many specific diagnoses.

In recent years, greater sensitivity and specificity of pericardioscopy than pericardial drainage or biopsy has been claimed, ${ }^{12}$ and it has been advocated as the preferred diagnostic technique. Although pericardioscopy may offer an advantage in selected patients, the available evidence does not seem to justify a widening of the indications for pericardial drainage. In addition to a likely selection bias, it is not clear whether a diagnosis relevant for management purposes could not be achieved by other means in most patients. Probably pericardioscopy should not modify the basic approach to the management of patients with pericardial effusion, although it may improve the diagnostic yield in selected patients or be the preferred method for drainage in experienced hands. 
Table 1 Prevalence of cardiac tamponade in acute pericardial disease

\begin{tabular}{lllll}
\hline & $\begin{array}{l}\text { Number of } \\
\text { patients }\end{array}$ & Tamponade & p Value \\
\hline $\begin{array}{l}\text { Neoplastic pericarditis } \\
\text { Tuberculous pericarditis }\end{array}$ & 26 & $16(61 \%)$ & \\
$\begin{array}{l}\text { Purulent pericarditis } \\
\text { Acute idiopathic pericarditis }\end{array}$ & 221 & $32(14 \%)$ & \\
\hline
\end{tabular}

Modified from Soler-Soler J, Permanyer-Miralda G, Sagristà-Sauleda J. A systematic diagnostic approach to primary acute pericardial disease: the Barcelona experience. In: Shabetai R, ed. Diseases of the pericardium. Cardiology Clinics 1990;8:639-44.

\section{DIAGNOSIS OF TUBERCULOUS AND NEOPLASTIC PERICARDITIS}

In our part of the world tuberculous pericarditis should not be diagnosed unless confirmed by microbiology or histology. Although the search for tubercle bacilli in pericardial fluid has a low yield, histology is more sensitive. ${ }^{12}$ The value of identifying tubercle bacilli in other sources (sputum, gastric aspirate or biopsy of large lymph nodes) cannot be overemphasised. ${ }^{12}$ For prognostic purposes a quick diagnosis is desirable, and thus one may ask whether high concentrations of adenosine deaminase in pleural or pericardial fluid should by themselves be sufficient diagnostic evidence. ${ }^{13}$ Our policy is to consider them just supportive rather than confirmative evidence of tuberculosis and to proceed with the search for mycobacteria or definite granulomas. On the other hand, low concentrations in pleural fluid should indeed not be taken as definitive evidence against pericardial tuberculosis. The diagnostic value of intermediate pericardial values $(30-70$ $\mathrm{iu} / \mathrm{l})$ is probably not well documented. Although it is likely that DNA amplification techniques in pericardial fluid may simplify the diagnosis, ${ }^{14}$ clinical experience is not sufficient for a definitive answer.

Primary or secondary neoplastic involvement of the pericardium may develop in a few patients without previously known malignancy, usually presenting with tamponade. ${ }^{3}$ Unlike in tuberculosis, here examination of the fluid has a larger yield than unguided biopsy techniques. ${ }^{12}$

\section{NEED FOR A COMPREHENSIVE APPROACH TO AETIOLOGIC DIAGNOSIS}

There is not enough evidence in the literature to give hard and fast rules for the aetiologic diagnosis in all cases of acute pericardial disease, and a systematic approach such as our own, which is based on an experience of more than two decades, seems justified. Essentially, our guideline ${ }^{7}{ }^{15}$ consists of three stages.

Stage 1 includes basic laboratory studies, chest $x$ ray, and Doppler echocardiogram. For patients without associated illness in whom clinical disease does not remit within one week or who have clinical features of tamponade, anti-DNA antibodies, rheumatoid factor, and three sputum or gastric aspirate cultures for mycobacteria are carried out. If definite pleural effusion is present, thoracocentesis is indicated. In addition to routine cytological and biochemical studies in pleural fluid, adenosine deaminase activity is measured and a search for mycobacteria is also done. Stage 1 also includes any investigation (such as computed tomographic scan, lymph node biopsy, serologic tests for toxoplasma, legionella, and mycoplasma, or assessment of the immunologic status) warranted by individual clinical findings.

Stage 2 involves pericardiocentesis, which is only indicated to treat cardiac tamponade and when purulent pericarditis is suspected. In pericardial fluid the same investigations as indicated for pleural fluid are performed. In appropriate laboratories, polymerase chain reaction (PCR) for tubercle bacilli can be measured.

Stage 3 involves subxiphoid pericardial drainage and biopsy, with histological examination (including stains for mycobacteria) and culture of the biopsy specimen. We indicate biopsy when pericardiocentesis is ineffective or tamponade relapses, and in selected patients with definite clinical disease of more than three weeks duration and no diagnosis. The decision for choosing pericardiocentesis rather than surgical drainage in the mentioned indications is based on local experience.

Our guideline showed good diagnostic performance in an initial study, ${ }^{3}$ and it has been validated in other settings. ${ }^{79}$ Despite its possible limitations, this approach—which could be modified depending on the frequency of particular disorders in other populations-provides a rational and adequate way to manage most patients with acute pericardial disease. Chronic pericardial effusion requires a different approach. $^{716}$

A relevant question is whether our guideline, or the principles underlying it, should be modified in patients with HIV infection. It seems tempting to enlarge the indications of pericardial drainage in these patients, as a wider spectrum of specific aetiologies has been suggested for their pericardial effusions. ${ }^{17}$ However, although further studies are needed, in most cases no specific aetiology is identified, ${ }^{18}$ and when it is, it may be of questionable clinical relevance because of the patient's condition. ${ }^{19}$ Therefore, while individualised attitudes may be warranted, for the time being there seems to be no reason to change a basically conservative approach to pericardial drainage in HIV patients, which should be indicated only to treat haemodynamic embarrassment or when there is a strong suspicion of treatable specific disease.

Correspondence to: Dr Gaietà Permanyer, Servei de Cardiologia, Hospital General Vall d'Hebron, Pg. Vall d'Hebron 119-129, 08035 Barcelona, Spain; gpermany@vhebron.net

\section{REFERENCES}

1 Nugue O, Millaire A, Porte $\mathrm{H}$, et al. Pericardioscopy in the etiologic diagnosis of pericardial effusion in 141 consecutive patients. Circulation 1996;94:1035-41.

2 Seferovic PM, Ristic AD, Maksimovic R, et al. Diagnostic value of pericardial biopsy: improvement with extensive sampling enabled by pericardioscopy. Circulation 2003;107:978-83.

3 Permanyer-Miralda G, Sagristà-Sauleda J, Soler-Soler J. Primary acute pericardial disease: a prospective series of 231 consecutive patients. Am J Cardiol 1985;56:623-30.

4 Permanyer-Miralda G, Sagristà-Sauleda J, Shabetai R, et al. Acute pericardial disease: an approach to etiologic diagnosis and treatment. In: Soler-Soler J, Permanyer-Miralda G, Sagristà-Sauleda J, eds. Pericardial disease: new insights and old dilemmas. Dordrecht: Kluwer Academic Publishers, 1990:193-214

5 Zayas R, Anguita M, Torres F, et al. Incidence of specific etiology and role of methods for specific etiologic diagnosis of primary acute pericarditis. Am J Cardiol 1995;75:378-82.

6 Mayosi BM, Nteskhe M, Volmink JA, et al. Interventions for treating tuberculous pericarditis. Cochrane Database Systematic Review 2002; CD000526.

7 Sagristá-Sauleda J, Mercé J, Permanyer-Miralda G, et al. Clinical clues to the causes of large pericardial ffusion. Am J Med 2000;109:95-101.

8 Atar S, Chiu J, Forrester JS, et al. Bloody pericardial effusion in patients with cardiac tamponade. Is the cause cancerous, tuberculous, or iatrogenic in the 1990s? Chest 1999;116:1564-9.

9 Sagristà-Sauleda J, Barrabés JA, Permanyer-Miralda G, et al. Purulent pericarditis: review of a 20 -year experience in a general hospital. J Am Coll Cardiol 1993;22:1661-5.

10 Mercé J, Sagristá-Sauleda J, Permanyer-Miralda G, et al. Should pericardial drainage be performed routinely in patients who have a large pericardial effusion without tamponade? Am J Med 1998;105:106-9.

11 Maisch B, Ristic AD, Pankuweit S. Intrapericardial treatment of autoreactive pericardial effusion with triamcinolone; the way to avoid side effects of systemic corticosteroid therapy. Eur Heart J 2002;23:1503-8.

12 Sagristà-Sauleda J, Permanyer-Miralda G, Soler-Soler J. Tuberculous pericarditis: ten year experience with a prospective protocol for diagnosis and treatment. J Am Coll Cardiol 1988; 1 1:724-8. 
13 Miller KD. Adenosine deaminase in pericardial fluids. Which test is best? Chest 1995; 108:1766

14 Cegielski JP, Devlin BH, Morris AJ, et al. Comparison of PCR, culture and histopathology for diagnosis of tuberculous pericarditis. J Clin Microbiol $1997 \cdot 35 \cdot 3254-7$

15 Service of Cardiology, Hospital General Vall d'Hebron, Barcelona. Appendix 1. Protocol for the diagnosis and management of pericardial diseases. In: Soler-Soler J, Permanyer-Miralda G, Sagristà-Sauleda J, eds. Pericardial disease: new insights and old dilemmas. Dordrecht: Kluwer Academic Publishers, 1990:217-22.
16 Sagristà-Sauleda J, Angel J, Permanyer-Miralda G, et al. Long-term follow-up of idiopathic chronic pericardial effusion. N Engl J Med 1999;341:2054-9.

17 Chen Y, Brennessel D, Walters J, et al. Human immunodeficiency virusassociated pericardial effusion: report of 40 cases and review of the literature. Am Heart J 1999; 137:516-21.

18 Heidenreich PA, Eisenberg MJ, Kee LL, et al. Pericardial effusion in AIDS. Incidence and survival. Circulation 1995;92:3229-34.

19 Flum DR, McGinn JR, Tyras DH. The role of the 'pericardial window' in AIDS. Chest 1995; 107:1522-5.

\section{IMAGES IN CARDIOLOGY}

\section{Aortic intramural haematoma leading to aortic dissection}

A ortic intramural haematoma (AIH), a variant form of aortic dissection, is characterised by absence of intimal tear and direct flow communication between the true and false lumen. Diagnostic criteria for AIH by transoesophageal echocardiography (TOE) include: (1) absence of dissection membrane; (2) no communication between the false and true lumen upon Doppler examination; (3) regional circular or crescentic thickening of the aortic wall $>0.7 \mathrm{~cm}$.

In AIH, wall morphology may change very rapidly. Bleeding into the aortic media may be self limiting, but may lead to classic dissection, as well as to

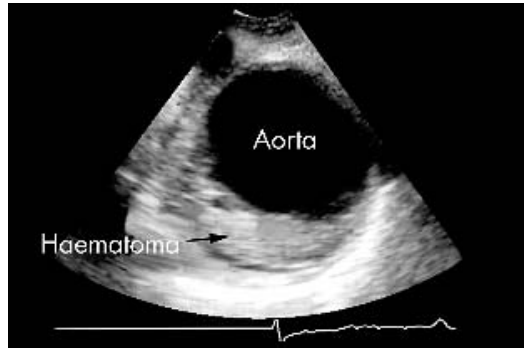

rapid aortic dilatation or circumferential and longitudinal extension of the haematoma.

A 64 year old hypertensive patient was admitted because of chest pain with radiation to the back. The ECG was normal, as were myocardial enzymes and ventricular wall motion assessed by transthoracic echocardiography. An aortic dissection was then suspected; the TOE (below left) revealed an $\mathrm{AIH}$, characterised by thickening $(>7 \mathrm{~mm})$ of the descending aorta. The haematoma extended for about $8 \mathrm{~cm}$.

The patient was monitored, and blood pressure was maintained as low as possible; symptoms disappeared in six

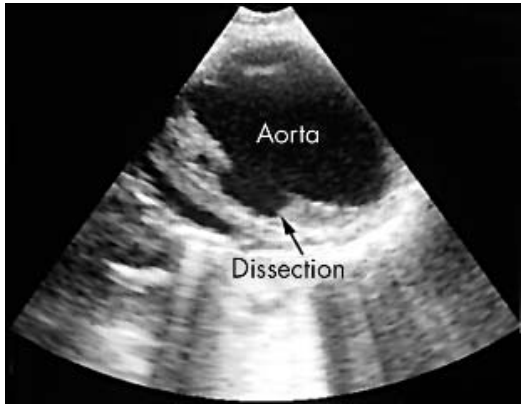

hours. Four days later, the chest pain reoccurred with the same characteristics. A new TOE (below centre) showed an aortic dissection, with a large tear, at the level of the previously observed AIH. A computed tomographic scan confirmed the dissection. An endoluminal aortic prosthesis was then applied, followed by complete resolution of the dissection (below right).

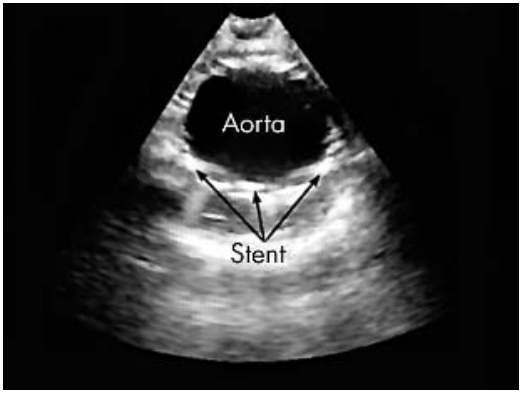

\section{ELECTRONIC PAGES}

\section{Heart Online case reports: www.heartjil.com}

 he follow electronic only articles are published in conjunction with this issue of Heart.

Evidence for "lumen sealing" with sirolimus eluting stents in the treatment of complex coronary artery dissection

E M Wong, C Pawsey, H C Lowe

Immediate and two month angiographic and intravascular ultrasound examination of sirolimus eluting stents deployed for complex coronary dissection is presented. The findings support the hypothesis that this novel treatment option is both effective and safe.

(Heart 2004;90:el3) www.heartjnl.com/cgi/content/full/90/ $3 / \mathrm{e} 13$
Clopidogrel induced urticarial rash in a patient with left main stem percutaneous coronary intervention: management issues

S K Khambekar, J Kovac, A H Gershlick

Clopidogrel, an ideal treatment for prevention of subacute stent thrombosis, may not be feasible to use in every patient. Ticlopidine (plus aspirin) is a very good alternative, although the risks of life threatening neutropenia should mandate regular monitoring of blood counts. It is proposed that patients undergoing angioplasty and stenting should carry a warning card in an effort to make the public and general practitioners aware that antiplatelet treatment after angioplasty plays an important part in ensuring successful outcome.

(Heart 2004;90:el4) www.heartjnl.com/cgi/content/full/90/ $3 / \mathrm{e} 14$ 